\title{
Une passion pour la médecine de famille ancrée dans le relief accidenté du Bouclier canadien
}

$\mathrm{D}^{\mathrm{re}}$ Cervin a grandi à Harrow, une petite communauté du Sud-Ouest ontarien. Adolescente, elle ne rêvait que d'une chose:partir à la découverte du monde. Mais ce sont deux aventures bien précises qui ont déclenché son indéfectible passion pour la médecine de famille rurale. D'abord, un congé sabbatique de son père, psychologue à l'Université de Windsor lui a permis, à l'âge de 16 ans, de voyager pendant un an aux États-Unis, en France, en Angleterre et en Espagne. La future $\mathrm{D}^{\mathrm{re}}$ Cervin avait alors 16 ans.

"J'ai pris la décision de devenir médecin durant cette année sabbatique en Europe, dit-elle. J'adorais les sciences et j'adorais travailler avec les gens. Mes parents m'avaient également transmis un sens aigu de la justice sociale. Je pensais que, si je devenais médecin, les gens accorderaient plus d'importance à ce que j'avais à dire."

L'année suivante, elle est partie avec deux amies à Sudbury, dans le Nord de l'Ontario. Voyant sous ses yeux s'incarner la beauté des peintures du Groupe des Sept, $\mathrm{D}^{\mathrm{re}}$ Cervin a immédiatement eu le coup de foudre. C'est à ce moment-là qu'elle a commencé à rêver de devenir médecin de famille en milieu rural. Elle s'est ensuite inscrite à la Faculté de médecine de l'Université de Toronto.

Ses deux mois de travail à Prince George, C.-B. avec un médecin suppléant permanent, ont été l'une des expériences les plus marquantes de sa formation. $\mathrm{D}^{\mathrm{re}}$ Cervin a travaillé dans tous les services de l'hôpital local - obstétrique, urgence et salle d'opération. Après un stage rotatoire à Toronto, elle a entrepris la deuxième année de sa résidence à l'Université Dalhousie de Halifax, N.-É. De là, elle a repris le chemin du Nord de l'Ontario.

$\mathrm{D}^{\mathrm{re}}$ Cervin a intégré la communauté médicale de Timmins, Ont., un endroit qui lui a semblé parfait pour ouvrir un cabinet. Dès qu'elle a pris conscience des problèmes et des besoins locaux, elle a voulu s'impliquer. C'est ainsi qu'elle a aidé à créer un groupe de santé des femmes qui organisait des conférences annuelles dans le Nord de l'Ontario auxquelles étaient invités des présentateurs de toutes les régions du pays.

"Ces conférences m'ont ouvert les yeux sur les injustices mondiales, la violence à l'égard des femmes et leur situation dans le monde. On commençait alors tout juste à ouvrir des centres d'aide et d'écoute et des maisons d'hébergement pour les femmes. Depuis, je nourris un grand intérêt pour la santé des femmes."

Au milieu des années 1980, D ${ }^{\text {re }}$ Cervin travaillait à Sault Ste Marie dans l'une des premières équipes de santé familiale de l'Ontario. Quand on lui a offert un poste de médecin de famille universitaire à l'Université Dalhousie, une fonction qui comportait un volet santé des femmes, elle a dû faire un choix difficile. Déménager avec un enfant en bas âge et abandonner une pratique qu'elle adorait représentait tout un défi. Mais l'appel de la médecine universitaire était irrésistible. La famille est donc partie pour la Nouvelle-Écosse et $\mathrm{D}^{\text {re }}$ Cervin est devenue directrice du service de médecine de famille de l'hôpital Grace Maternity.

En 1997, elle a été nommée directrice du programme de résidence en médecine de famille de l'Université Dalhousie. C'est à ce moment-là que l'éducation médicale est devenue son centre d'intérêt principal et qu'elle a décidé de faire une maîtrise en éducation. Sa thèse portait sur les facteurs qui influencent la décision des résidents en médecine de famille de dispenser des soins complets et globaux.

$\mathrm{D}^{\mathrm{re}}$ Cervin est retournée dans le Nord en 2011 quand on lui a offert le poste de doyenne associée responsable de la formation postdoctorale à l'École de médecine du Nord de l'Ontario. La possibilité qu'on lui offrait signifiait qu'elle devrait partager sa vie familiale entre Sudbury et Halifax puisque son conjoint, $\mathrm{D}^{\mathrm{r}}$ David Gass, était lié aux Maritimes par des liens familiaux et professionnels $\mathrm{D}^{\mathrm{re}}$ Cervin occupe maintenant le poste de vice-doyenne à l'enseignement de l'École de médecine du Nord de

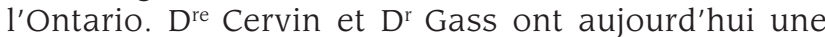
famille recomposée qui compte cinq enfants adultes et quatre petits-enfants.

Être sollicitée pour occuper des postes de direction a été une constante dans la vie de $\mathrm{D}^{\text {re }}$ Cervin. Elle s'est impliquée au sein du CMFC en 1997 quand elle a été recrutée par la Section des enseignants. Elle a été présidente du Bureau des examens et de la certification et de la Fondation pour la recherche et l'éducation (aujourd'hui la Fondation pour l'avancement de la médecine familiale). Elle s'est jointe au Conseil d'administration du CMFC en 2018 et se dit ravie d'aborder son mandat de présidente du CMFC.

"Une des choses que j'ai apprises au cours des dernières années en siégeant au Conseil d'administration $c^{\prime}$ est que le soutien aux membres et la représentation de leurs intérêts et de leurs besoins doivent faire partie des objectifs du président ou de la présidente. Ainsi, mes objectifs personnels importent peu; l'important, c'est ma capacité d'écouter les médecins de famille du Canada, de répondre à leurs besoins et de soutenir leurs priorités, tout en gardant à l'esprit la justice sociale et l'équité pour tous les Canadiens et Canadiennes." 